\title{
The phytohormone abscisic acid increases triacylglycerol content in the green microalga Chlorella saccharophila (Chlorophyta)
}

\author{
Patricia Yolanda Contreras-Pool ${ }^{1}$, Santy Peraza-Echeverria ${ }^{1}$, Ángela Francisca Ku-González ${ }^{2}$ \\ and Virginia Aurora Herrera-Valencia ${ }^{1, *}$ \\ ${ }^{1}$ Unidad de Biotecnología, Centro de Investigación Científica de Yucatán (CICY), Calle 43 No. 130 x 32 y 34, \\ Col. Chuburná de Hidalgo, C.P. 97205, Mérida, Yucatán, México \\ ${ }^{2}$ Unidad de Bioquímica y Biología Molecular de Plantas, Centro de Investigación Científica de Yucatán (CICY), \\ Calle 43 No. 130 x 32 y 34, Col. Chuburná de Hidalgo, C.P. 97205, Mérida, Yucatán, México
}

\begin{abstract}
Microalgae are currently a very promising source of biomass and triacylglycerol (TAG) for biofuels. In a previous study, we identified Chlorella saccharophila as a suitable source of oil for biodiesel production because it showed high biomass and lipid content with an appropriate fatty acid methyl esters profile. To improve the TAG accumulation in C. saccharophila, in this study we evaluated the effect of abscisic acid (ABA) addition on cell concentration, lipid content and TAG production in this microalga. First, we evaluated the effects of four ABA concentrations $(1,4,10$, and $20 \mu \mathrm{M})$ added at the beginning of a single-stage cultivation strategy, and found that all concentrations tested significantly increased cell concentration and TAG content in C. saccharophila. We then evaluated the addition of $1 \mu \mathrm{M}$ ABA during the second stage of a two-stage cultivation strategy and compared it with a nitrogen deficiency treatment (ND) and a combination of ND and ABA (ND + ABA). Although ABA alone significantly increased lipid and TAG contents compared with the control, ND showed significantly higher TAG content, and ND + ABA showed the highest TAG content. When comparing the results of both strategies, we found a superior response in terms of TAG accumulation with the addition of $1 \mu \mathrm{M} \mathrm{ABA}$ at the beginning of a single-stage cultivation system. This strategy is a simple and effective way to improve the TAG content in $C$. saccharophila and probably other microalgae as a feedstock for biodiesel production.
\end{abstract}

Key Words: abscisic acid; Chlorella saccharophila; green microalgae; lipids; phytohormone; triacylglycerol

Abbreviations : ABA, abscisic acid; DBW, dry biomass weight; FAME, fatty acid methyl esters; ND, nitrogen deficiency treatment; TAG, triacylglycerol; TAP, Tris-acetate-phosphate; TAP-N, TAP medium lacking $\mathrm{NH}_{4} \mathrm{Cl}$

\section{INTRODUCTION}

Currently, microalgal biomass is considered one of the most promising and environmentally friendly sources of triacylglycerol (TAG) for biodiesel production; however, there are still some challenges that need to be overcome before large-scale production of microalgal biomass and oil for biofuels can become a reality (Rawat et al. 2013, Vanthoor-Koopmans et al. 2013, Medipally et al. 2015). Biomass and lipid content are two very important factors to improve to make microalgal culture commercially feasible (Medipally et al. 2015). Therefore, several strategies
(9) $\$$ This is an Open Access article distributed under the terms of the Creative Commons Attribution Non-Commercial License (http://creativecommons.org/licenses/by-nc/3.0/) which permits unrestricted non-commercial use, distribution, and reproduction in any medium, provided the original work is properly cited.
Received June 22, 2016, Accepted September 3, 2016

* Corresponding Author

E-mail: vicky@cicy.mx

Tel: +52-999-9428330, Fax: +52-999-9813900 
have been investigated to improve TAG accumulation in microalgae; for example, alkaline $\mathrm{pH}$ stress, light intensity, and nutrient starvation (Guckert and Cooksey 1990, Mujtaba et al. 2012, Sun et al. 2014). In this regard, nitrogen deficiency is generally considered the most successful approach to enhance lipid accumulation, particularly TAGs, in green microalgae (Rawat et al. 2013); however, this increase in lipids is generally accompanied by a decrease in biomass. Genetic engineering has also been suggested as an alternative to improve microalgal biomass and lipid production, and although very promising, this technology is still being developed or is undeveloped in most microalgal species (Medipally et al. 2015). Other strategies involve the use of biochemical stimulants, including phytohormones, to increase biomass and lipid content (Hunt et al. 2010, Park et al. 2013). Hunt et al. (2010) evaluated 12 biochemical stimulants and discovered phytohormones from the auxin family, mainly 1-naphthaleneacetic acid, had a positive effect on the biomass productivity of Chlorella sorokiniana. Phytohormones have been found in several microalgae (Tarakhovskaya et al. 2007, Lu and Xu 2015) and although little is known about their functions, the exogenous addition of these molecules can have positive metabolic effects in these microorganisms; for example, abscisic acid (ABA) has been shown to improve tolerance to dehydration, oxidative and salinity stresses as well as nitrogen deprivation in a number of microalgae (reviewed by Lu and Xu 2015). Recently, Park et al. (2013) evaluated the effects of several phytohormones on cell density, concentration of chlorophyll, protein and starch, as well as fatty acid methyl esters (FAME) yield in the green microalga Chlamydomonas reinhardtii, and found that the addition of $10 \mathrm{ppm} \mathrm{ABA}(37.83 \mu \mathrm{M})$ during the lag phase significantly increased FAME yield but decreased cell density.

In a previous study, our research group proposed Chlorella saccharophila (Krüger) Migula, a green microalgae native to the Yucatan Peninsula in Mexico, as a promising source of oil for biofuel production because of its high biomass, lipid content and productivity, in addition to its suitable fatty acid profile for biodiesel (Herrera-Valencia et al. 2011). To improve TAG accumulation without decreasing biomass in this microalga, we evaluated the effect of exogenous ABA application on cell concentration, lipid and TAG contents in C. saccharophila.

\section{MATERIALS AND METHODS}

\section{Microalgal strain and culture conditions}

Chlorella saccharophila (UADY-PRIORI-014-FMVZ-04) (Herrera-Valencia et al. 2011). was maintained on Tris-acetate-phosphate (TAP) (Harris 1989) under axenic conditions, at $25 \pm 2^{\circ} \mathrm{C}$ under a $16 \mathrm{~h} / 8 \mathrm{~h}$ (light / dark) cycle at a light intensity of $90 \mu \mathrm{mol} \mathrm{m}{ }^{-2} \mathrm{~s}^{-1}$ on a rotary shaker (140 rpm). Two culture media were used during this study: complete TAP medium containing $104.8 \mathrm{mg} \mathrm{L}^{-1}$ nitrogen, and TAP medium lacking $\mathrm{NH}_{4} \mathrm{Cl}$ as a nitrogen source (TAP-N), therefore containing only $0.68 \mathrm{mg} \mathrm{L}^{-1}$ nitrogen from $\mathrm{Mo}_{7} \mathrm{O}_{24}\left(\mathrm{NH}_{4}\right)_{6} \cdot 4 \mathrm{H}_{2} \mathrm{O}$ in trace metals. The stock solution of ABA (Sigma-Aldrich, St. Louis, MO, USA) was dissolved in $1 \mathrm{M} \mathrm{KOH}$ and then $100 \mathrm{~mL}$ of distilled water was added. To evaluate the effect of ABA on cell number, dry biomass weight (DBW), lipid, and TAG accumulation in C. saccharophila, a single-stage cultivation strategy was used in which exponentially growing cells were inoculated into $250 \mathrm{~mL}$ Erlenmeyer flasks containing $50 \mathrm{~mL}$ liquid TAP medium with an initial cell concentration of 10,000 cells $\mathrm{mL}^{-1}$, and were supplemented with $\mathrm{ABA}$ at one of four ABA concentrations $(1,4,10$, or $20 \mu \mathrm{M}$ dissolved in distilled water; distilled water was added to the control cells). The cultures were grown for 10 days and collected by centrifugation for further analysis. We also evaluated the effect of ABA supplementation in combination with nitrogen deficiency. To achieve this, a two-stage cultivation strategy was employed in which cultures were inoculated as described above and after 10 days the cells were harvested by centrifugation, transferred to fresh TAP with the appropriate experimental treatment, grown for 7 days and collected by centrifugation for further analysis. Three experimental treatments were evaluated: (1) ABA, TAP supplemented with $1 \mu \mathrm{M} \mathrm{ABA}$; (2) ND, TAP medium lacking $\mathrm{NH}_{4} \mathrm{Cl}$ as a nitrogen source (TAP-N); (3) ND + ABA, TAP-N supplemented with $1 \mu \mathrm{M}$ ABA. Fresh TAP was used as a control. All experiments were carried out in triplicate, and each experiment was carried out twice. One-way ANOVA and Fisher's least significant difference test were used to determine significant differences among means at the $5 \%$ level of significance (Statgraphics Plus 4.1). All reagents and solvents were analytical grade.

\section{Growth evaluation}

Cellular concentration was determined at the end of the experiments using a Neubauer hemacytometer (Hausser Scientific, Horsham, USA) and a Primo Star mi- 

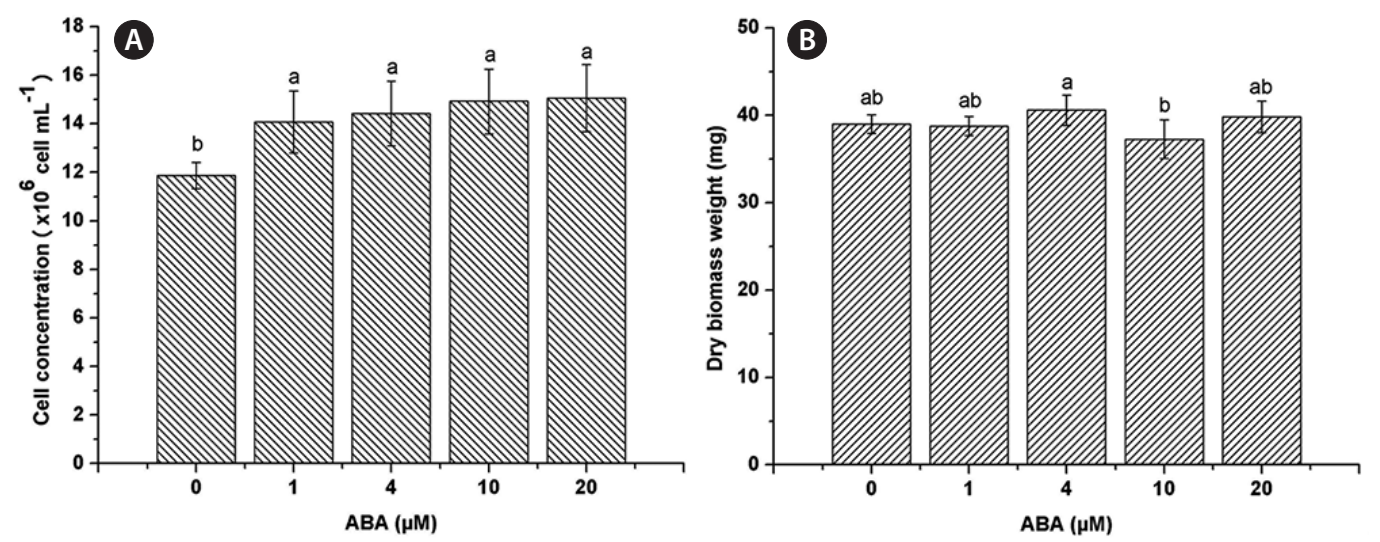

Fig. 1. Effect of abscisic acid (ABA) on Chlorella saccharophila growth. Cell concentration (A) and dry biomass weight (B) of $C$. saccharophila. Cells were incubated in Tris-acetate-phosphate supplemented with different concentrations of ABA for 10 days. Each value is the mean \pm standard deviation $(n=3)$. Different lowercase letters indicate significant difference between means (one-way ANOVA, Fisher's least significant difference test, $\mathrm{p}<0.05)$.

croscope (Carl Zeiss, Oberkochen, Germany). The cells were harvested by centrifugation and dried using a freeze dryer for $48 \mathrm{~h}$. The resulting pellet was weighed to get the DBW. Pellets were stored at $-80^{\circ} \mathrm{C}$ for further lipid and TAG analysis.

\section{Lipid and TAG content}

Lipids were extracted from the microalgal biomass using a dry extraction procedure with a chloroform: methanol $(2: 1, \mathrm{v} / \mathrm{v})$ solvent mixture according to Widjaja et al. (2009). The solvent mixture was separated from the biomass and evaporated to obtain the lipid extract. The procedure was repeated twice to achieve complete lipid extraction. The TAG content in the total extractable lipids was determined as described previously by Arias-Forero et al. (2013) using Triglicéridos Liquicolor GPO-PAP (Stanbio Laboratory, Boerne, TX, USA) according to the manufacturer's protocol.

\section{Confocal imaging of live cells}

Cells were stained with Nile Red (1 $\mathrm{mg} \mathrm{mL}^{-1}$ final concentration; Sigma-Aldrich) as described by Wang et al. (2009) and Cakmak et al. (2012). Images were acquired using an LSCM FV 1000 laser scanning confocal microscope (Olympus, Tokyo, Japan) and a Plan UPLFLN 40 oil immersion objective lens with a numerical aperture of 1.30. The Nile Red signal was captured using a laser excitation line at $488 \mathrm{~nm}$, and the emission was collected at $510 \mathrm{~nm}$; chlorophyll fluorescence was captured using a laser excitation line at $633 \mathrm{~nm}$, and the emission was collected at $650 \mathrm{~nm}$. Images were merged and pseudocolored using the FV 10 ASW 4.1 CLSM Olympus viewer software (Olympus).

\section{RESULTS}

\section{Effect of ABA on Chlorella saccharophila growth}

C. saccharophila reached a cell concentration of 11.88 $\pm 0.54 \times 10^{6}$ cells $\mathrm{mL}^{-1}$ on TAP medium (control). Cell concentration was positively affected by ABA under all the concentrations evaluated, $1 \mu \mathrm{M}\left(14.07 \pm 1.28 \times 10^{6}\right.$ cells $\left.\mathrm{mL}^{-1}\right), 4 \mu \mathrm{M}\left(14.43 \pm 1.34 \times 10^{6}\right.$ cells $\left.\mathrm{mL}^{-1}\right), 10 \mu \mathrm{M}(14.93 \pm$ $1.33 \times 10^{6}$ cells $\left.\mathrm{mL}^{-1}\right)$, and $20 \mu \mathrm{M}\left(15.05 \pm 1.38 \times 10^{6}\right.$ cells $\mathrm{mL}^{-1}$ ) (Fig. 1A). For DBW, there was no significant difference between any of the ABA concentrations tested (1 $\mu \mathrm{M}, 38.77 \pm 1.1 \mathrm{mg} ; 4 \mu \mathrm{M}, 40.6 \pm 1.73 \mathrm{mg} ; 10 \mu \mathrm{M}, 37.27$ $\pm 2.22 \mathrm{mg} ; 20 \mu \mathrm{M}, 39.83 \pm 1.82 \mathrm{mg}$; control, $39 \pm 1.08 \mathrm{mg}$ ) (Fig. 1B).

\section{Effect of ABA on Chlorella saccharophila lipid content and TAG accumulation}

The lipid extract was $20.03 \pm 3.76 \mathrm{mg}$ for the control and similar amounts were found for all ABA concentrations tested, $1 \mu \mathrm{M}(20.63 \pm 3.25 \mathrm{mg}), 4 \mu \mathrm{M}(21.77 \pm 1.82$ $\mathrm{mg}), 10 \mu \mathrm{M}(21.33 \pm 2.85 \mathrm{mg})$, and $20 \mu \mathrm{M}(22.83 \pm 5.33$ mg) (Fig. 2A). The lipid content of C. saccharophila as a percentage of dry biomass weight (\% DBW) was calculated, and no significant differences were found between the control (51.24 $\pm 8.27 \%)$ and any of the ABA treatments 

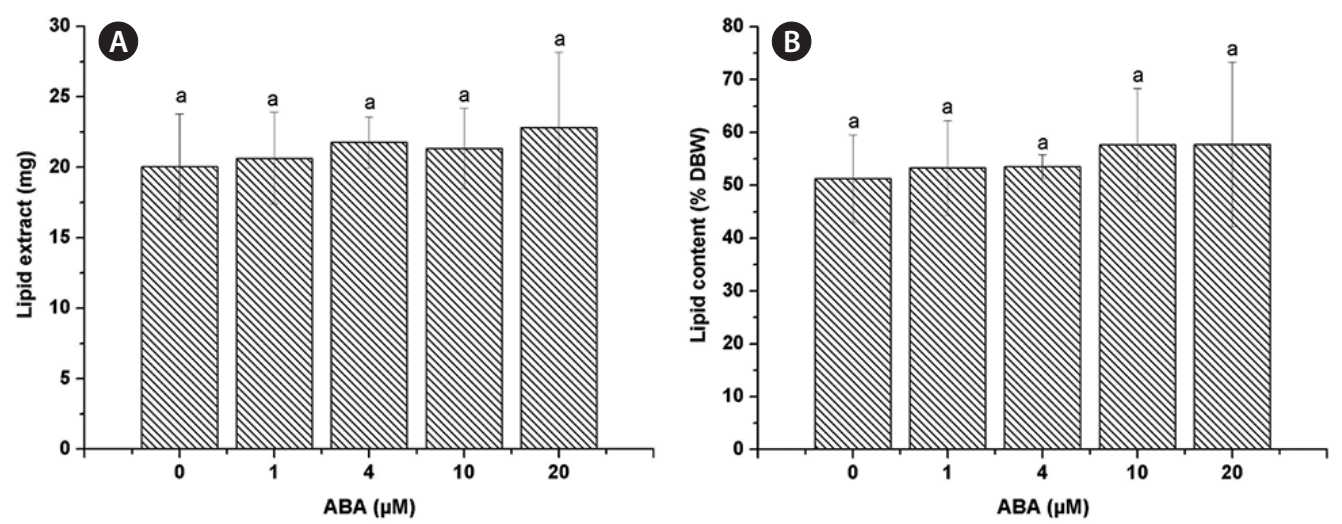

Fig. 2. Effect of abscisic acid (ABA) on Chlorella saccharophila lipid content. Lipid extract $(A)$ and lipid content as a percentage of dry biomass weight (\% DBW) (B) of C. saccharophila. Cells were incubated in Tris-acetate-phosphate supplemented with different concentrations of ABA for 10 days. Each value is the mean \pm standard deviation $(n=3)$. Different lowercase letters indicate significant difference between means (one-way ANOVA, Fisher's least significant difference test, $p<0.05)$.
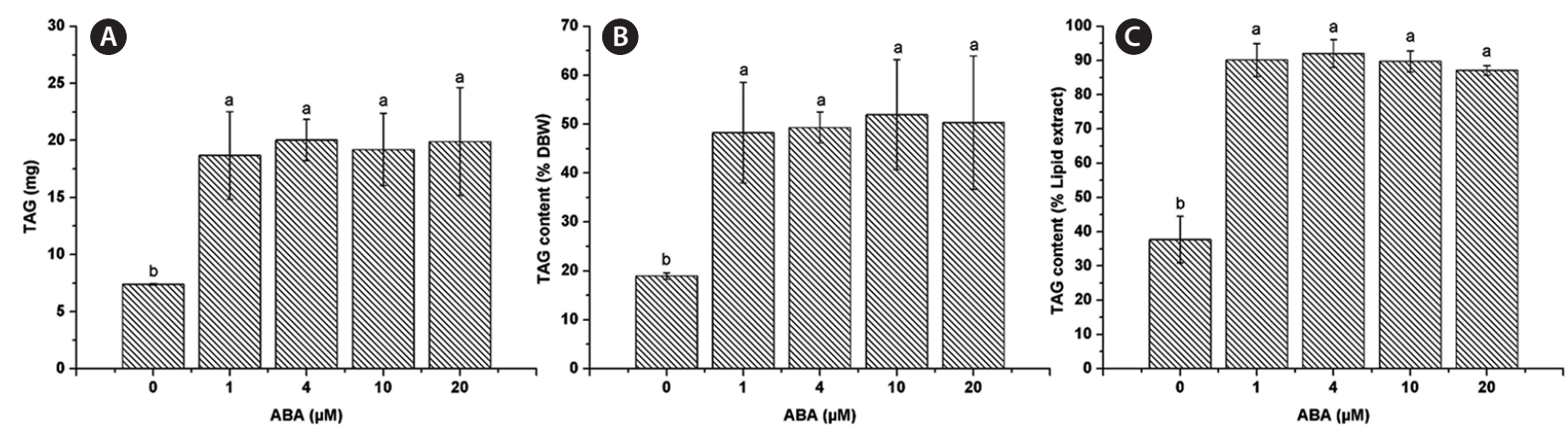

Fig. 3. Effect of abscisic acid (ABA) deficiency on Chlorella saccharophila triacylglycerol (TAG) accumulation. TAG accumulation (A), TAG content as a percentage of dry biomass weight (\% DBW) (B), and TAG content as a percentage of lipid extract (C) of $C$. saccharophila. Cells were incubated in Tris-acetate-phosphate supplemented with different concentrations of ABA for 10 days. Each value is the mean \pm standard deviation $(n=3)$. Different lowercase letters indicate significant difference between means (one-way ANOVA, Fisher's least significant difference test, $\mathrm{p}<0.05$ ).

tested $(1 \mu \mathrm{M}, 53.28 \pm 8.94 \% ; 4 \mu \mathrm{M}, 53.55 \pm 2.24 \% ; 10 \mu \mathrm{M}$, $57.64 \pm 10.70 \%$; $20 \mu \mathrm{M}, 57.74 \pm 15.57 \%$ ) (Fig. 2B). Regarding TAG accumulation in C. saccharophila, the TAG content was $7.39 \pm 0.07 \mathrm{mg}$ in the control. Notably, a significant increase in TAG accumulation was obtained with all the ABA concentrations tested, $1 \mu \mathrm{M}(18.70 \pm 3.83 \mathrm{mg}), 4$ $\mu \mathrm{M}(20.04 \pm 1.81 \mathrm{mg}), 10 \mu \mathrm{M}(19.20 \pm 3.17 \mathrm{mg})$, and $20 \mu \mathrm{M}$ $(19.91 \pm 4.72 \mathrm{mg})$ (Fig. 3A). We then calculated the TAG content as \% DBW, which was $18.96 \pm 0.64 \%$ DBW in the control. Again, significantly higher values were found for all the ABA concentrations tested, $1 \mu \mathrm{M}(48.27 \pm 10.29 \%$ DBW), $4 \mu \mathrm{M}$ ( $49.31 \pm 3.18 \%$ DBW), $10 \mu \mathrm{M}(51.95 \pm 11.26 \%$ DBW), and $20 \mu \mathrm{M}(50.33 \pm 13.63 \% \mathrm{DBW})$ (Fig. 3B). We also calculated TAG content as a percentage of the lipid extract (\% lipid extract). The control had $37.75 \pm 6.82 \%$ lipid ex- tract, while significantly higher values were found for all the ABA concentrations tested $(1 \mu \mathrm{M}, 90.13 \pm 4.85 \%$; 4 $\mu \mathrm{M}, 92.06 \pm 4.03 \% ; 10 \mu \mathrm{M}, 89.74 \pm 3.06 \%$; and $20 \mu \mathrm{M}, 87.11$ $\pm 1.14 \%$ ) (Fig. 3C).

\section{Effect of the combination of ABA and nitrogen deficiency on Chlorella saccharophila growth}

C. saccharophila reached a cell concentration of 11.46 $\pm 0.41 \times 10^{6}$ cells $\mathrm{mL}^{-1}$ on TAP medium (control). Cell concentration was positively affected by $\mathrm{ND}+\mathrm{ABA}$ treatment $\left(12.76 \pm 0.69 \times 10^{6}\right.$ cells $\left.\mathrm{mL}^{-1}\right)$, while ABA treatment $(12.06 \pm$ $0.8810^{6}$ cells $\left.\mathrm{mL}^{-1}\right)$ showed no significant difference compared with the control, and ND treatment $(9.82 \pm 0.38 \times$ $10^{6}$ cells $\mathrm{mL}^{-1}$ ) caused a significant decrease in microalgal 

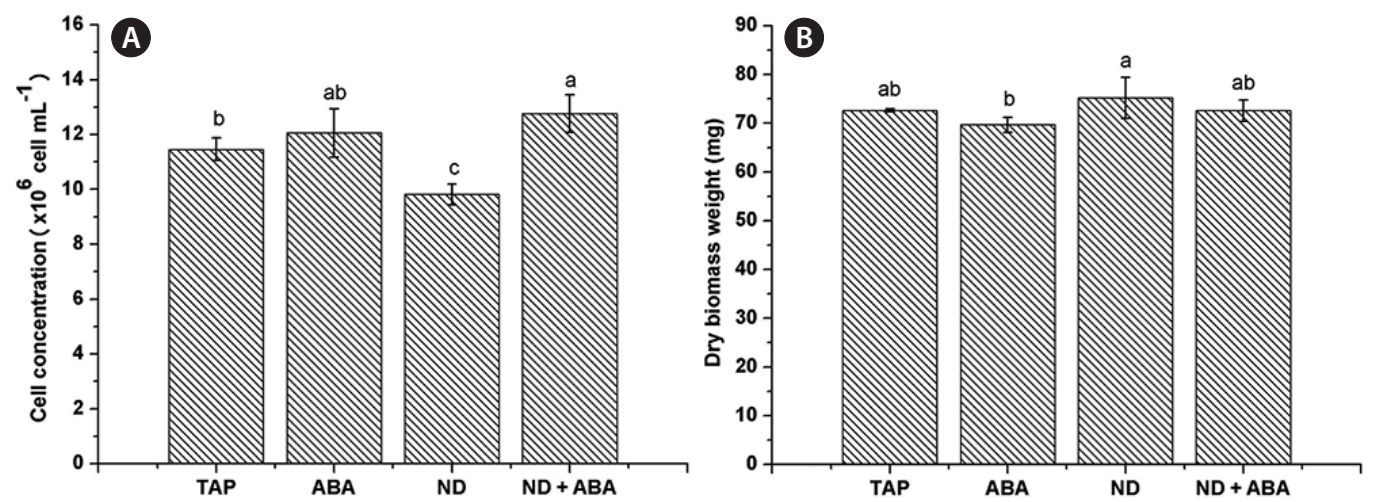

Fig. 4. Effect of the combination of abscisic acid (ABA) and nitrogen deficiency on Chlorella saccharophila growth. Cell concentration (A) and dry biomass weight (B). Cells were incubated in Tris-acetate-phosphate (TAP) medium for 10 days, and then harvested by centrifugation and transferred to fresh medium for 7 days. TAP, complete TAP medium as a control; ABA, TAP medium supplemented with $1 \mu$ M ABA; ND, TAP with nitrogen deficiency; ND + ABA, TAP with nitrogen deficiency supplemented with $1 \mu \mathrm{M} A B A$. Each value is the mean \pm standard deviation $(n=3)$. Different lowercase letters indicate significant difference between means (one-way ANOVA, Fisher's least significant difference test, $\mathrm{p}<0.05$ ).
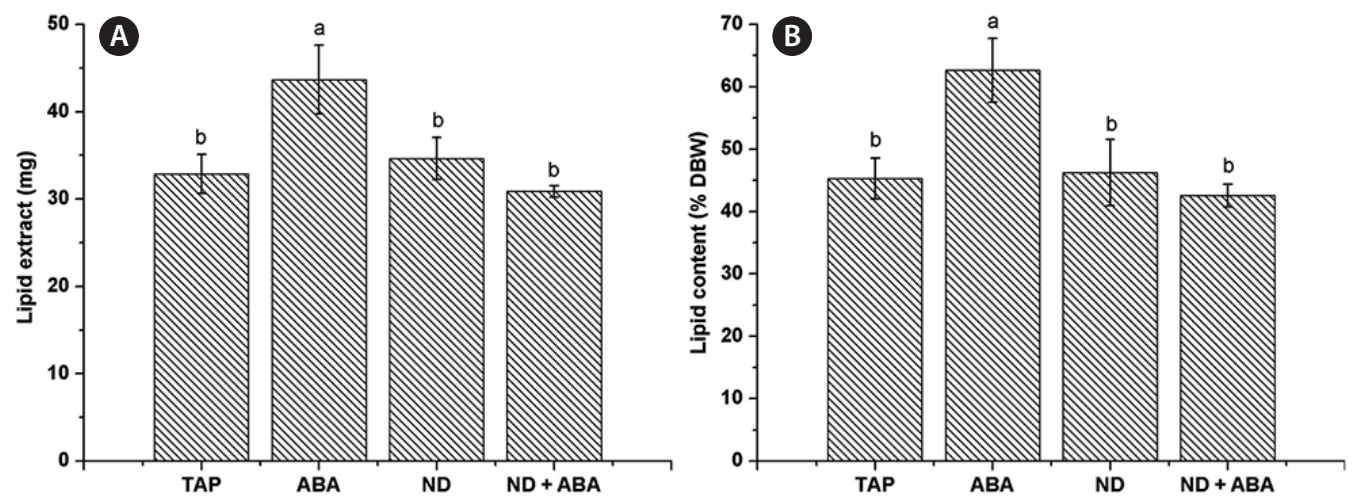

Fig. 5. Effect of the combination of abscisic acid (ABA) and nitrogen deficiency on Chlorella saccharophila lipid content. Lipid extract (A) and lipid content as a percentage of dry biomass weight (\% DBW) (B). Cells were incubated in Tris-acetate-phosphate (TAP) medium for 10 days, and then harvested by centrifugation and transferred to fresh medium for 7 days. TAP, complete TAP medium as a control; ABA, TAP medium supplemented with $1 \mu \mathrm{M} A B A$; ND, TAP with nitrogen deficiency; ND + ABA, TAP with nitrogen deficiency supplemented with $1 \mu M$ ABA. Each value is the mean \pm standard deviation $(n=3)$. Different lowercase letters indicate significant difference between means (one-way ANOVA, Fisher's least significant difference test, $\mathrm{p}<0.05$ ).

cell concentration (Fig. 4A). For DBW, none of the treatments tested, $\mathrm{ABA}(69.67 \pm 1.55 \mathrm{mg})$, ND $(75.2 \pm 4.2 \mathrm{mg})$, or ND + ABA (72.57 $\pm 2.18 \mathrm{mg})$, showed significant differences compared with the control (72.6 $\pm 0.3 \mathrm{mg}$ ) (Fig. 4B).

\section{Effect of the combination of $A B A$ and nitrogen deficiency on Chlorella saccharophila lipid con- tent and TAG accumulation}

A significantly higher amount of lipid extract was obtained from the ABA treatment ( $43.67 \pm 3.95 \mathrm{mg}$ ), while
$\mathrm{ND}(34.63 \pm 2.4 \mathrm{mg})$ and $\mathrm{ND}+\mathrm{ABA}(30.87 \pm 0.65 \mathrm{mg})$ showed no significant difference compared with the control (32.87 $\pm 2.25 \mathrm{mg}$ ) (Fig. 5A). The lipid content of C. saccharophila as \% DBW was calculated; the ABA treatment showed a significantly higher amount $(62.66 \pm 5.12 \%)$, while there were no significant differences among the ND treatment $(46.24 \pm 5.32 \%)$, the $\mathrm{ND}+\mathrm{ABA}$ treatment $(42.57$ $\pm 1.81 \%$ ) and the control (45.28 $\pm 3.25 \%$ ) (Fig. $5 \mathrm{~B})$. The amount of TAG accumulated was $5.09 \pm 0.56 \mathrm{mg}$ in the control. Interestingly, a significant increase in TAG accumulation was obtained with all the treatments evaluated, 

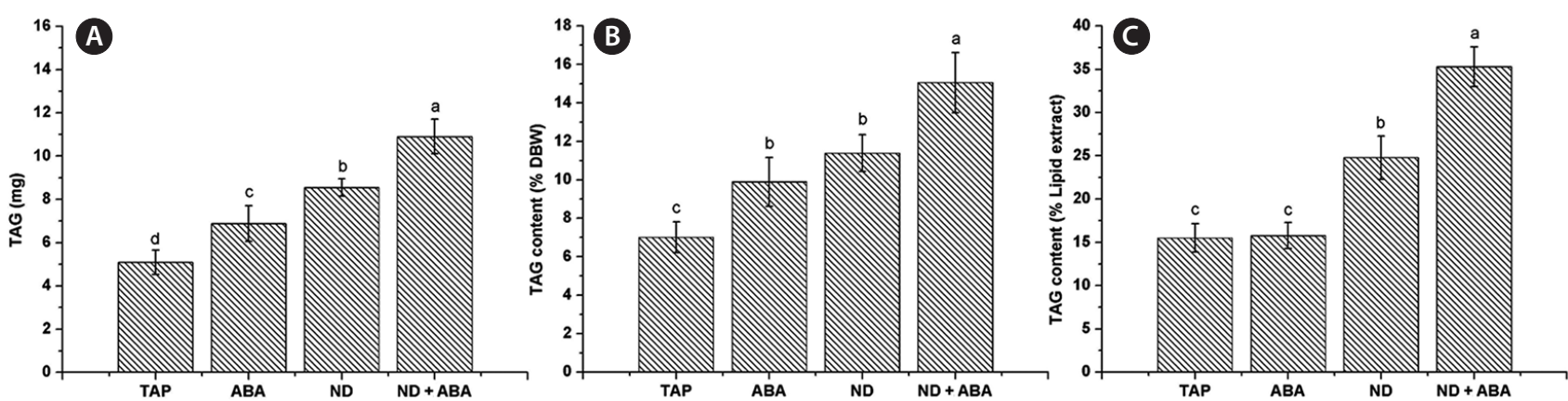

Fig. 6. Effect of the combination of abscisic acid (ABA) and nitrogen deficiency on Chlorella saccharophila triacylglycerol (TAG) accumulation. TAG accumulation (A), TAG content as a percentage of dry biomass weight (\% DBW) (B), and TAG content as a percentage of lipid extract (C) of $C$. saccharophila. Cells were incubated in Tris-acetate-phosphate (TAP) medium for 10 days, and then harvested by centrifugation and transferred to fresh medium for 7 days. TAP, complete TAP medium as a control; ABA, TAP medium supplemented with $1 \mu M$ ABA; ND, TAP with nitrogen deficiency; ND + ABA, TAP with nitrogen deficiency supplemented with $1 \mu \mathrm{M} A B A$. Each value is the mean \pm standard deviation $(n=3)$. Different lowercase letters indicate significant difference between means (one-way ANOVA, Fisher's least significant difference test, $p<0.05$ ).

$\mathrm{ABA}(6.88 \pm 0.83 \mathrm{mg}), \mathrm{ND}(8.54 \pm 0.41 \mathrm{mg})$, and $\mathrm{ND}+\mathrm{ABA}$ $(10.9 \pm 0.80 \mathrm{mg})$ (Fig. 6A). We calculated the TAG content as a percentage of $\%$ DBW, which was $7.01 \pm 0.79 \%$ DBW in the control. Again, significantly higher values were found for all the treatments evaluated (ABA, $9.87 \pm 1.26 \%$ DBW; ND, $11.39 \pm 0.95 \%$ DBW; ND + ABA, $15.05 \pm 1.56 \%$ DBW) (Fig. 6B). We also calculated the TAG content as a percentage of lipid extract (\% lipid extract) and found that it was $15.05 \pm 1.64 \%$ lipid extract in the control. Although ABA showed no significant difference (15.78 $\pm 1.49 \%$ lipid extract), the ND (24.77 $\pm 2.52 \%$ lipid extract) and ND + $\mathrm{ABA}(35.31 \pm 2.3 \%$ lipid extract) treatments showed significantly higher values (Fig. 6C).

\section{Confocal microscopy analysis}

Since there were no significant differences among the ABA treatments evaluated during the single-stage cultivation strategy, we chose the lowest concentration of ABA (1 $\mu \mathrm{M})$ for confocal microscopy analysis. Living C. saccharophila cells were stained with Nile Red, a reagent that yields brilliant yellow fluorescence in a neutral lipid environment (Greenspan et al. 1985, Cooksey et al. 1987). The confocal image (Fig. 7A) shows the presence of brilliant yellow color produced by the interaction of Nile Red with neutral lipids in C. saccharophila cells. The confocal analysis allowed us to visualize the neutral lipids contained in C. saccharophila cells and to qualitatively detect an increase in these lipids in cultures with $1 \mu \mathrm{M}$ ABA treatment compared with the control without ABA.

The confocal microscopy analysis also allowed us to qualitatively observe the increase in neutral lipids in
C. saccharophila cultures with $\mathrm{ABA}, \mathrm{ND}$, and $\mathrm{ND}+\mathrm{ABA}$ treatments compared with the control, evaluated using the two-stage cultivation strategy (Fig. 7B).

\section{DISCUSSION}

In the present study, we were interested in evaluating the effect of ABA addition on growth and lipid accumulation in C. saccharophila. We first evaluated four different ABA concentrations $(1,4,10$ and $20 \mu \mathrm{M})$ added at the beginning of the culture, and found that all concentrations tested significantly increased cell concentration but not DBW or lipid content. Additionally, we found that all ABA concentrations tested were able to increase TAG accumulation in C. saccharophila compared with the control, both as a $\%$ DBW and as a percentage of lipid extract. This result indicates that while ABA-treated cells synthesized an amount of lipids similar to the control, they mostly accumulated lipids in the form of TAG. Remarkably, ABA caused an increase in cell number without affecting DBW, which was unexpected since ABA is known to inhibit cell division in plants (Stals and Inzé 2001). The addition of similar concentrations of ABA to the medium was evaluated in another green microalga, C. reinhardtii, and no changes in growth (measured as cell density, $\mathrm{g} \mathrm{L}^{-1}$ ) were found compared with the control; however, cell number was not measured (Park et al. 2013). Furthermore, in $C$. reinhardtii, the addition of $500 \mu \mathrm{M}$ ABA to the medium caused an increase in the growth of cells, measured as mg of dry weight $\mathrm{mL}^{-1}$, compared with the control (Yoshida et al. 2003). When using chlorophyll content as an indicator 


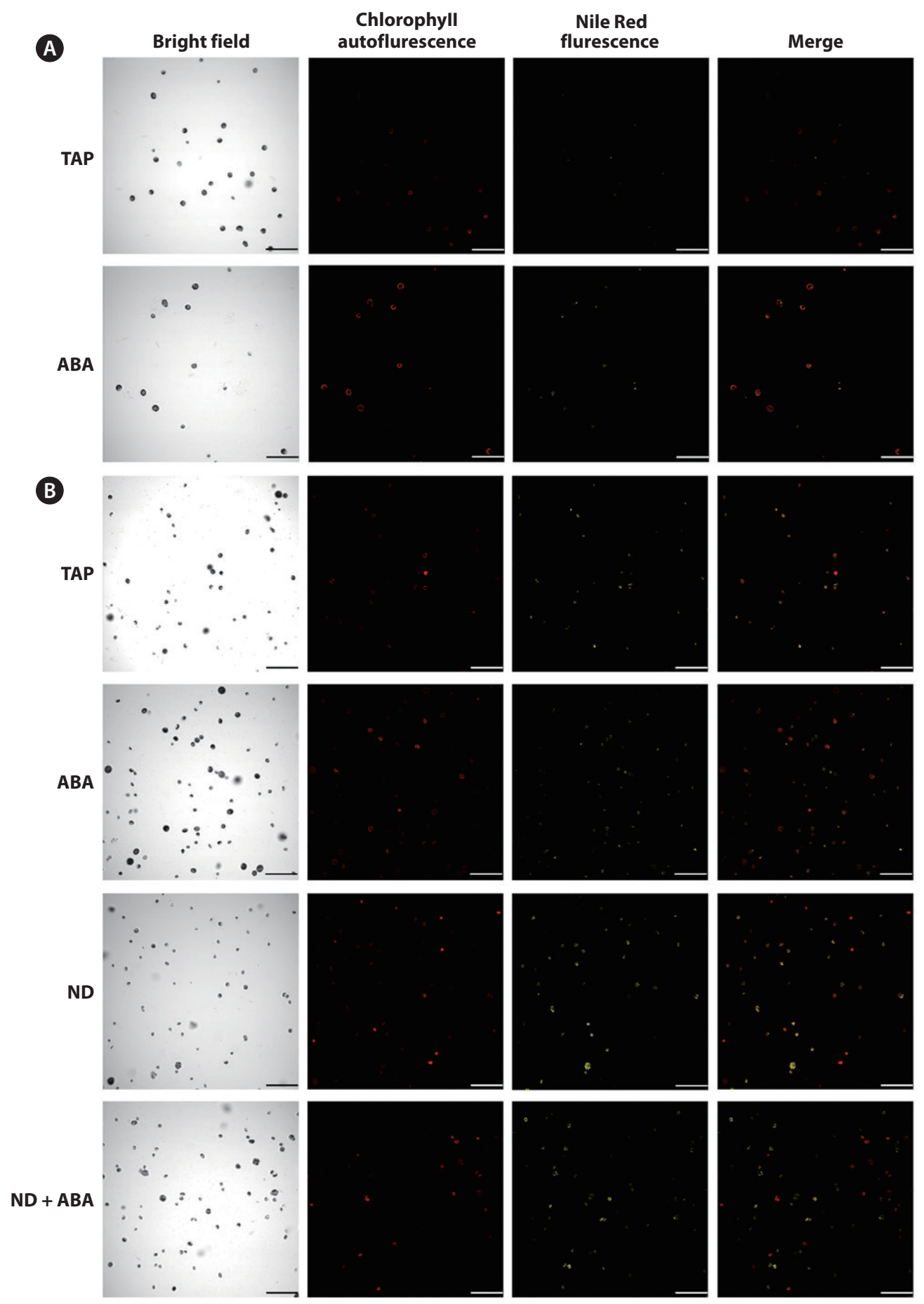

Fig. 7. Confocal microscopy of Chlorella saccharophila. (A) Cells were incubated in Tris-acetate-phosphate (TAP) alone or supplemented with $1 \mu \mathrm{M}$ abscisic acid (ABA) for 10 days. (B) Cells were incubated in TAP medium for 10 days, and then harvested by centrifugation and transferred to fresh medium for 7 days. TAP, complete TAP medium as a control; ABA, TAP medium supplemented with $1 \mu M$ ABA; ND, TAP with nitrogen deficiency; ND + ABA, TAP with nitrogen deficiency supplemented with $1 \mu \mathrm{M} A B A$. Scale bars represent: A \& B, $50 \mu \mathrm{m}$. 
of cell growth in this microalga, $500 \mu \mathrm{M}$ ABA also caused an increase in cell growth in the presence or absence of osmotic and salt stress (Yoshida et al. 2004).

Exogenous ABA has been reported to cause lipid accumulation in isolated plant embryos and plant cell suspensions (Rodriguez-Sotres and Black 1994, Kharenko et al. 2010). Interestingly, the ABA treatments evaluated in $C$. reinhardtii by Park et al. (2013) caused a 10-13\% increase of FAME yield, which is an indirect measure of TAG accumulation in cells. Notably, in our study, the response of C. saccharophila to ABA led to a higher increase in TAG accumulation; more than double the total TAG and TAG as a percentage of DBW, and double or more the TAG as a percentage of lipid extract. This result supports the use of ABA to increase TAG in microalgal culture, and also supports our previous claim that C. saccharophila is an attractive and suitable source of TAG for biodiesel (HerreraValencia et al. 2011). However, more research is needed to understand the specific role of ABA in lipid accumulation, particularly TAG, in microalgae.

In a previous study by our research group, we used a two-stage cultivation system to evaluate the effect of nitrogen depletion on cell growth, lipid accumulation and lipid profile in C. saccharophila (Herrera-Valencia et al. 2011), and found that nitrogen deficiency increased lipid accumulation and FAME yield, and that the fatty acid profile was suitable for biodiesel production. In this study, we evaluated ABA addition, nitrogen deficiency and a combination of both, applied at the second stage of a twostage cultivation system. When only ABA was applied in this manner, and contrary to what we found in the singlestage culture, there was no significant difference in cell number compared with the control. A possible explanation is that at the time ABA was applied during the second stage, the biomass had already accumulated and cell division was not very active. Therefore, it was surprising to find that while there was a significant decrease in cell number under the ND treatment (as expected), when ABA was applied in combination with ND an increase in cell number was observed compared with the control and the ND treatment. A possible explanation could be that the combined effect of both treatments induced cell division in some manner yet to be determined.

Contrary to what we found when ABA was applied at the beginning of the single-stage culture, there was a significant increase in total lipid content when the ABA treatment was applied at the second stage of the twostage cultivation strategy, compared with the control and the other treatments. This increase corresponded to an increase in total TAG content and TAG as a percent- age of DBW, but not TAG as a percentage of lipid extract, suggesting that the TAG increase was due to an increase in total lipids. ND treatment significantly increased TAG content compared with the control, as expected. Remarkably, all measurements of TAG content were significantly higher in the $\mathrm{ND}+\mathrm{ABA}$ treatment than in all the other treatments including ND alone, suggesting that ND as a stress and ABA as a stress signal had a combined effect that led the cells to accumulate more TAG. There have been a few reports on the physiological roles of endogenous ABA in green microalgae. For example, endogenous ABA increased significantly in both hypertonic and hypotonic environments in Dunaliella sp. and under nitrogen deficiency in Dunaliella sp. and Nannochloropsis oceanica (Tominaga et al. 1993, Lu et al. 2014). Kobayashi et al. (1997) suggested that ABA may act as a stress hormone in the morphogenesis of Haematococcus pluvialis, since endogenous ABA biosynthesis was induced by active oxygen species and exogenous ABA caused the recovery of algal encystment under drought stress even in the presence of the encystment inhibitor chloramphenicol. Furthermore, it has been reported that ABA acts as a signal that enhances tolerance to oxidative and osmotic stress in C. reinhardtii, probably by helping to reduce oxidative damage in cells derived from exposure to stress (Yoshida et al. 2003, 2004).

Several strategies have been used to increase lipid content and TAG accumulation in microalgae, and nitrogen depletion has been an effective way to achieve this (Yoon et al. 2015). A two-stage cultivation strategy has been one of the most successful strategies to date for increasing TAG content in microalgal cell cultures (Rodolfi et al. 2009, Ho et al. 2010, Sun et al. 2014). This strategy involves efficient biomass accumulation during nutrientcomplete conditions in the first stage and then collection and resuspension of the cells in a nutrient-limited fresh medium, particularly nitrogen limited, for lipid accumulation. However, its application at the industrial scale is not yet feasible, since it is costly, time consuming and requires centrifugation, which is not practical. Several modifications have been attempted to improve this strategy. Recently, Fan et al. (2014) demonstrated the feasibility of using a trophic transition cultivation mode for lipid production, which consists of heterotrophy-nitrogen deprivation during a two-stage production method. More recently, Li et al. (2015) proposed two-stage N-deficient cultivation of microalgae without centrifugation as a suitable, effective and low energy consumption method for lipid accumulation in Scenedesmus sp. Alternatively, the addition of phytohormones to the culture medium has 
been proposed as an attractive strategy to increase lipid production. For example, Park et al. (2013) proposed the use of phytohormones, including ABA, to increase biomass and FAME yield, thereby reducing the production costs of biofuels from microalgae, using nitrogen-limited media and C. reinhardtii as a model system.

When we compared the results of the two strategies evaluated in this study ABA addition during a single-stage cultivation and ND + ABA during a two-stage cultivation we found that the highest levels (double or more) of TAG as a percentage of DBW and of lipid extract were obtained with the addition of $1 \mu \mathrm{M} \mathrm{ABA}$ at the beginning of a single-stage culture, which is a less time-consuming and laborious cultivation strategy. Taking our results together, we propose the addition of $1 \mu \mathrm{M} \mathrm{ABA}$ at the beginning of a single-stage culture as an effective and simple way to improve TAG accumulation in C. saccharophila. This strategy might be useful in other oleaginous microalgae to improve the accumulation of TAG as a feedstock for biodiesel production.

\section{ACKNOWLEDGEMENTS}

This research was supported by the Consejo Nacional de Ciencia y Tecnología (CONACYT, México) Project No. 169217. Patricia Yolanda Contreras Pool received support from CONACYT (Scholarship No. 224389). The authors thank Ileana C. Borges Argáez for technical support.

\section{REFERENCES}

Arias-Forero, D., Hayashida, G., Aranda, M., Portilla, T., García, A. \& Díaz-Palma, P. 2013. Protocol for maximizing the triglycerides-enriched lipids production from $\mathrm{Du}$ naliella salina SA32007 biomass, isolated from the Salar de Atacama (Northern Chile). Adv. Biosci. Biotechnol. 4:830-839.

Cakmak, T., Angun, P., Demiray, Y. E., Ozkan, A. D., Elibol, Z. \& Tekinay, T. 2012. Differential effects of nitrogen and sulfur deprivation on growth and biodiesel feedstock production of Chlamydomonas reinhardtii. Biotechnol. Bioeng. 109:1947-1957.

Cooksey, K. E., Guckert, J. B., Williams, S. A. \& Callis, P. R. 1987. Fluorometric determination of the neutral lipid content of microalgal cells using Nile Red. J. Microbiol. Methods 6:333-345.

Fan, J., Cui, Y., Wan, M., Wang, W. \& Li, Y. 2014. Lipid accumulation and biosynthesis genes response of the oleagi- nous Chlorella pyrenoidosa under three nutrition stressors. Biotechnol. Biofuels 7:17.

Greenspan, P., Mayer, E. P. \& Fowler, S. D. 1985. Nile red: a selective fluorescent stain for intracellular lipid droplets. J. Cell Biol. 100:965-973.

Guckert, J. B. \& Cooksey, K. E. 1990. Triglyceride accumulation and fatty acid profile changes in Chlorella (Chlorophyta) during high $\mathrm{pH}$-induced cell cycle inhibition. J. Phycol. 26:72-79.

Harris, E. H. 1989. The Chlamydomonas sourcebook: a comprehensive guide to biology and laboratory use. Academic Press, San Diego, CA, 780 pp.

Herrera-Valencia, V. A., Contreras-Pool, P. Y., López-Adrián, S. J., Peraza-Echeverría, S. \& Barahona-Pérez, L. F. 2011. The green microalga Chlorella saccharophila as a suitable source of oil for biodiesel production. Curr. Microbiol. 63:151-157.

Ho, S. -H., Chen, W. -M. \& Chang, J. -S. 2010. Scenedesmus obliquus $\mathrm{CNW}-\mathrm{N}$ as a potential candidate for $\mathrm{CO}_{2}$ mitigation and biodiesel production. Bioresour. Technol. 101:8725-8730.

Hunt, R. W., Chinnasamy, S., Bhatnagar, A. \& Das, K. C. 2010. Effect of biochemical stimulants on biomass productivity and metabolite content of the microalga, Chlorella sorokiniana. Appl. Biochem. Biotechnol. 162:2400-2414.

Kharenko, O. A., Zaharia, L. I., Giblin, M., Čekić, V., Taylor, D. C., Don Palmer, C., Abrams, R. C. \& Loewen, M. C. 2010. Abscisic acid metabolism and lipid accumulation of a cell suspension culture of Lesquerella fendleri. Plant Cell Tissue Organ Cult. 105:415-422.

Kobayashi, M., Hirai, N., Kurimura, Y., Ohigashi, H. \& Tsuji, Y. 1997. Abscisic acid-dependent algal morphogenesis in the unicellular green alga Haematococcus pluvialis. Plant Growth Regul. 22:79-85.

Li, X., Wang, M., Liao, X., Chen, H., Dai, Y. \& Chen, B. 2015. Two stages of N-deficient cultivation enhance the lipid content of microalga Scenedesmus sp. J. Am. Oil Chem. Soc. 92:503-512.

Lu, Y., Tarkowská, D., Turečková, V., Luo, T., Xin, Y., Li, J., Wang, Q., Jian, N., Strnad, M. \& Xu, J. 2014. Antagonistic roles of abscisic acid and cytokinin during response to nitrogen depletion in oleaginous microalga Nannochloropsis oceanica expand the evolutionary breadth of phytohormone function. Plant J. 80:52-68.

Lu, Y. \& Xu, J. 2015. Phytohormones in microalgae: a new opportunity for microalgal biotechnology? Trends Plant Sci. 20:273-282.

Medipally, S. R., Yusoff, F. M., Banerjee, S. \& Shariff, M. 2015. Microalgae as sustainable renewable energy feedstock for biofuel production. Biomed. Res. Int. 2015:519513. 
Mujtaba, G., Choi, W., Lee, C. -G. \& Lee, K. 2012. Lipid production by Chlorella vulgaris after a shift from nutrientrich to nitrogen starvation conditions. Bioresour. Technol. 123:279-283.

Park, W. -K., Yoo, G., Moon, M., Kim, C. W., Choi, Y. -E. \& Yang, J. -W. 2013. Phytohormone supplementation significantly increases growth of Chlamydomonas reinhardtii cultivated for biodiesel production. Appl. Biochem. Biotechnol. 171:1128-1142.

Rawat, I., Ranjith Kumar, R., Mutanda, T. \& Bux, F. 2013. Biodiesel from microalgae: a critical evaluation from laboratory to large scale production. Appl. Energy 103:444467.

Rodolfi, L., Chini Zittelli, G., Bassi, N., Padovani, G., Biondi, N., Bonini, G. \& Tredici, M. R. 2009. Microalgae for oil: strain selection, induction of lipid synthesis and outdoor mass cultivation in a low-cost photobioreactor. Biotechnol. Bioeng. 102:100-112.

Rodriguez-Sotres, R. \& Black, M. 1994. Osmotic potential and abscisic acid regulate triacylglycerol synthesis in developing wheat embryos. Planta 192:9-15.

Stals, H. \& Inzé, D. 2001. When plant cells decide to divide. Trends Plant Sci. 6:359-364.

Sun, X., Cao, Y., Xu, H., Liu, Y., Sun, J., Qiao, D. \& Cao, Y. 2014. Effect of nitrogen-starvation, light intensity and iron on triacylglyceride/carbohydrate production and fatty acid profile of Neochloris oleoabundans HK-129 by a twostage process. Bioresour. Technol. 155:204-212.

Tarakhovskaya, E. R., Maslov, Y. I. \& Shishova, M. F. 2007. Phy- tohormones in algae. Russ. J. Plant Physiol. 54:163-170.

Tominaga, N., Takahata, M. \& Tominaga, H. 1993. Effects of $\mathrm{NaCl}$ and $\mathrm{KNO}_{3}$ concentrations on the abscisic acid content of Dunaliella sp. (Chlorophyta). Hydrobiologia 267:163-168.

Vanthoor-Koopmans, M., Wijffels, R. H., Barbosa, M. J. \& Eppink, M. H. M. 2013. Biorefinery of microalgae for food and fuel. Bioresour. Technol. 135:142-149.

Wang, Z. T., Ullrich, N., Joo, S., Waffenschmidt, S. \& Goodenough, U. 2009. Algal lipid bodies: stress induction, purification, and biochemical characterization in wildtype and starchless Chlamydomonas reinhardtii. Eukaryot. Cell 8:1856-1868.

Widjaja, A., Chien, C. -C. \& Ju, Y. -H. 2009. Study of increasing lipid production from fresh water microalgae Chlorella vulgaris. J. Taiwan Inst. Chem. Eng. 40:13-20.

Yoon, S. Y., Hong, M. E., Chang, W. S. \& Sim, S. J. 2015. Enhanced biodiesel production in Neochloris oleoabundans by a semi-continuous process in two-stage photobioreactors. Bioprocess Biosyst. Eng. 38:1415-1421.

Yoshida, K., Igarashi, E., Mukai, M., Hirata, K. \& Miyamoto, K. 2003. Induction of tolerance to oxidative stress in the green alga, Chlamydomonas reinhardtii, by abscisic acid. Plant Cell Environ. 26:451-457.

Yoshida, K., Igarashi, E., Wakatsuki, E., Miyamoto, K. \& Hirata, K. 2004. Mitigation of osmotic and salt stresses by abscisic acid through reduction of stress-derived oxidative damage in Chlamydomonas reinhardtii. Plant Sci. 167:1335-1341. 